\title{
I Design of the Primary Prevention Parameters Evaluation (PREPARE) trial of implantablecardioverter defibrillators to reduce patient morbidity [NCT00279279]
}

\author{
Bruce L Wilkoff* ${ }^{\dagger 1}$, Richard Stern ${ }^{\dagger 2}$, Brian Williamson ${ }^{\dagger 3}$, Mark Wathen ${ }^{\dagger 4}$, \\ Keith Holloman ${ }^{\dagger 5}$, Ann Fieberg ${ }^{\dagger 5}$ and Mark Brown ${ }^{\dagger 5}$
}

Address: ${ }^{1}$ The Cleveland Clinic Lerner College of Medicine, Case Western Reserve University, Cleveland, OH, USA, ${ }^{2}$ Doctors Medical Center, San Pablo, CA, USA, ${ }^{3}$ William Beaumont Hospital, Troy, MI, USA, ${ }^{4}$ Vanderbilt University, Nashville, TN, USA and ${ }^{5}$ Medtronic, Inc, Minneapolis, MN, USA

Email: Bruce L Wilkoff* - wilkofb@ccf.org; Richard Stern - richardsternmd@yahoo.com; Brian Williamson - bwilliamson@mhgpc.com; Mark Wathen - mark.wathen@vanderbilt.edu; Keith Holloman - keith.holloman@medtronic.com;

Ann Fieberg - ann.m.fieberg@medtronic.com; Mark Brown - mark.l.brown@medtronic.com

* Corresponding author †Equal contributors

Published: 25 May 2006

Trials 2006, 7:18 doi:10.1186/1745-6215-7-18

This article is available from: http://www.trialsjournal.com/content/7/I//8

(C) 2006 Wilkoff et al; licensee BioMed Central Ltd.

This is an Open Access article distributed under the terms of the Creative Commons Attribution License (http://creativecommons.org/licenses/by/2.0), which permits unrestricted use, distribution, and reproduction in any medium, provided the original work is properly cited.

\begin{abstract}
Background: Implantable Cardioverter Defibrillator (ICD) therapy has been proven to be beneficial and efficacious for the treatment of serious ventricular tachyarrhythmias in primary prevention patients. However, primary prevention patients appear to have a lower incidence of ventricular arrhythmias in comparison to secondary prevention patients and consequently likely experience a higher proportion of detections due to supraventricular arrhythmias. Recent trials have demonstrated that strategic and specific programming choices reduce the number of inappropriate shocks and that anti-tachycardia pacing (ATP) is an effective alternative to shock therapy for many sustained ventricular arrhythmias.

Methods: The Primary Prevention Parameters Evaluation (PREPARE) study is a multi-center cohort study, evaluating the efficacy of a pre-specified strategic profile of VT/VF detection and therapy settings in 700 primary prevention patients in an effort to safely reduce the number of shock therapies delivered. The patients, both with and without cardiac resynchronization therapy, are compared to a well-qualified set $(n=691)$ of historical controls derived from the MIRACLE ICD and EMPIRIC trials. This manuscript describes the design of the PREPARE study. The study results, to be presented separately, will characterize the efficacy of this programming set (PREPARE) compared with physician-tailored programming (MIRACLE ICD and EMPIRIC).
\end{abstract}

\section{Background}

Implantable defibrillator therapy terminates ventricular tachyarrhythmias and improves survival of patients in multiple populations at risk of sudden cardiac death. [13] Initially, ICD therapy was applied only to secondary prevention patients: that is, those who had been resusci- tated from a cardiac arrest or other sustained ventricular tachyarrhythmias. The impact of other studies, such as MADIT, MUSTT, MADIT II and SCD-HeFT, as well as the CMS national coverage decision in January of 2005, is that the large majority of patients currently receiving ICDs 
have primary prevention indications and no symptomatic arrhythmias prior to ICD implantation. [4-8]

There are substantial differences in the clinical and rhythm characteristics of patients with primary and secondary prevention indications for ICD therapy. However, it is an important goal to minimize the morbidity of the therapy for all ICD patients. Patients who receive multiple shocks have difficulty adjusting to the ICD implantation, due to increased anxiety and depression. $[9,10]$ In addition, patients who have not had a significant life-threatening arrhythmic experience such as sudden cardiac death may be less accepting of unnecessary shock therapies.

The EMPIRIC and Painfree Rx studies have shown that a standardized set of VT/VF parameters extensively utilizing SVT discriminators and anti-tachycardia pacing (ATP) therapies can be effective in reducing patient shocks after ICD implantation for a mixed population of primary and secondary prevention patients. $[11,12]$ It is the purpose of the PREPARE trial to evaluate strategies specifically designed to reduce shocks in a population of patients with primary prevention ICD indications with and without cardiac resynchronization indications and therapy.

\section{Methods/Design Hypothesis}

The PREPARE study examines the hypothesis that a prespecified strategic profile of tachyarrhythmia detection and therapy parameters is able to reduce the overall morbidity of ICD therapy in patients with primary prevention ICD indications with or without cardiac resynchronization therapy.

\section{Primary endpoint}

Shock related morbidity is measured by the Morbidity Index and expressed through the incidence density. However, the reduction of shocked episodes in primary prevention patients via the use of a specific programming profile is useful only if it does not result in an increase in arrhythmogenic syncope and untreated sustained symptomatic VT/VF events. The Morbidity Index measures the benefit of reducing the number of shocks while also accounting for the possible side effects of 1) treating only faster tachyarrhythmias (cycle-lengths greater than 330 $\mathrm{ms}$ ) and 2) using a longer programmed delay before treating ventricular tachyarrhythmias with defibrillation.

\section{Morbidity index}

The components of the Morbidity Index are:

- Syncope (secondary to tachyarrhythmia or presumed tachyarrhythmia)

- Untreated sustained symptomatic VT/VF events
- Episodes of spontaneous ventricular tachyarrhythmia that result in device-delivered shock

- Episodes that result in an inappropriate device-delivered shock.

Incidence Density (ID)

The Morbidity Index incidence density is calculated by dividing the total number of Morbidity Index events by the total number of years of follow-up. The primary endpoint is the difference in Morbidity Index incidence density between the study patients programmed to the PREPARE settings and the control population programmed to physician-tailored programming.

\section{Secondary endpoint}

Morbidity Tachycardia Index

The key secondary endpoint is the difference in Morbidity Tachycardia Index incidence density, which adds antitachycardia pacing events to the Morbidity Index as a reflection of all tachycardia events, between patients programmed to the PREPARE settings and the control population.

\section{Design}

The PREPARE study is a prospective, single arm, multicenter cohort study designed to evaluate the efficacy of a pre-specified strategic profile of VT and VF detection and therapy settings designed for ICD indicated patients with no history of spontaneous sustained symptomatic VT or VF. All study patients received a Medtronic Marquis family ICD system with or without capacity for cardiac resynchronization implanted between October 2003 and April 2005. Approximately 700 patients were enrolled in the United States and the Netherlands and were programmed to the PREPARE parameters and followed for endpoints for 12 months after PREPARE programming.

For the purposes of this study, primary prevention patients are defined as those patients indicated for ICD or CRT + ICD implantation without a baseline history of ventricular fibrillation, ventricular flutter, monomorphic VT, polymorphic VT, or Torsades de Pointes. Patients may have a history of cardiovascular syncope, since the PREPARE findings are intended to be generalized to the primary prevention patient population seen in clinical practice.

- Inclusion criteria required:

OInitial implantation for primary prevention ICD indications or

O Prior ICD implantation within 6 months without subsequent 
spontaneous VT/VF episodes

- Patients were excluded for:

History of spontaneous sustained symptomatic ventricular arrhythmias,

An electrophysiology test in the past, with sustained inducible $\mathrm{VT}<180 \mathrm{bpm}$,

OAny ICD implanted greater than 6 months prior to the study,

An ICD implanted within the previous 6 months, with subsequent history of a spontaneous episode of VT or VF appropriately treated with either ATP or shock.

\section{Heart transplant}

Mechanical right heart valve.

\section{Control population}

The control population consists of the prospectively collected shock, tachycardia and event data combined from two randomized clinical ICD trials; the MIRACLE ICD Trial[13] and the EMPIRIC Trial. [11] Both of these trials included both primary and secondary prevention patients, but only the primary prevention patients were included in the control population for the PREPARE analysis.

Originally, the control population included only the primary prevention patient data from the MIRACLE ICD Trial. The MIRACLE ICD Trial was a multi-center randomized trial of 978 CRT-ICD patients, designed to assess the safety and effectiveness of biventricular CRT in primary and secondary prevention patients. However, in contrast to the MIRACLE ICD Trial which enrolled only patients receiving a cardiac resynchronization defibrillator (CRT+ICD), the PREPARE Study includes primary prevention patients implanted with either an ICD or CRT+ICD device. After an interim review of baseline characteristics during the enrollment phase of the PREPARE Trial, it became evident that $65 \%$ of the patient population did not require a cardiac resynchronization device. Therefore, the PREPARE Trial population was comprised of patients with more favorable clinical characteristics than the advanced heart failure patients enrolled in the MIRACLE ICD Trial. This imbalance threatened to falsely skew the results of the study, making it difficult to discern whether the potential efficacy of the PREPARE parameters would be the result of the recruitment of patients into the PREPARE study with less severe disease.
To address the imbalanced baseline characteristics between the PREPARE patients and the original control population, the primary prevention patients from the physician-tailored arm of the EMPIRIC study were combined with MIRACLE ICD control patients to produce the control population.

The EMPIRIC study was a multi-center randomized trial of 900 ICD patients, designed to compare standardized vs. physician-tailored VT/VF programming in both primary and secondary prevention patients. [11] The study was recently completed, and its patient population is thought to better reflect the higher ratio of ICD implant indications seen during enrollment into the PREPARE study than the MIRACLE ICD Trial, reported in 2003. [13] Additionally, there were a large number of patients (276) randomized in the EMPIRIC Trial to the physician-tailored arm who met the PREPARE Trial definition of primary prevention. These patients, along with the 415 primary prevention patients from the MIRACLE ICD Trial, have been combined, producing a total of 691 patients in the historical control sample.

When compared with PREPARE patient baseline demographics, the patient characteristics of the combined physician-tailored arm are not clinically different. The baseline clinical data for the study and control patients is listed in Table 1. This table includes baseline demographics which were collected in PREPARE, MIRACLE ICD and EMPIRIC. Demographics for MIRACLE ICD and EMPIRIC are shown separately and in combination.

\section{Programming}

The strategies for reducing shock morbidity are to 1) reduce over-treatment of self-terminating tachycardias, 2) reduce the mis-identification of supraventricular arrhythmias as ventricular arrhythmias, and 3) terminate ventricular tachycardia with anti-tachycardia pacing as frequently as possible. Since the patients enrolled in this trial had never experienced a sustained ventricular arrhythmia, the parameters were chosen to not treat: 1) slow and presumably less symptomatic tachycardias, and 2) faster but self-terminating arrhythmias.

The exact tachycardia detection and therapy parameters for the PREPARE study are listed in Table 2. In summary two therapy zones are programmed, treating all rhythms of cycle length less than $330 \mathrm{~ms}$ that meet the duration criteria of 30 of 40 intervals. A single anti-tachycardia burst is provided for tachycardias between 330 and $240 \mathrm{~ms}$. If the rhythm persists after ATP, a shock is provided. Rhythms faster than 240 ms receive shocks as the first therapy. A monitor zone is provided for tachycardia between 360 and 330 ms. Many SVT discriminators are programmed $\mathrm{ON}$. 
Table I: Patient Demographics

\begin{tabular}{|c|c|c|c|c|}
\hline & $\begin{array}{l}\text { PREPARE } \\
\text { Patients } \\
(\mathbf{N}=700)\end{array}$ & $\begin{array}{l}\text { MIRACLE } \\
\text { ICD Patients } \\
(\mathbf{N}=415)\end{array}$ & $\begin{array}{l}\text { EMPIRIC } \\
\text { Patients } \\
(\mathbf{N}=276)\end{array}$ & $\begin{array}{l}\text { Combined Control (MIRACLE } \\
\text { ICD and EMPIRIC) Patients } \\
(\mathrm{N}=691)\end{array}$ \\
\hline \multicolumn{5}{|l|}{ Gender (N, \%) } \\
\hline Male & 555 (79.3\%) & 310 (74.7\%) & 222 (80.4\%) & 532 (77\%) \\
\hline Female & $145(20.7 \%)$ & $105(25.3 \%)$ & $54(19.6 \%)$ & $159(23 \%)$ \\
\hline \multicolumn{5}{|l|}{ Age (years) } \\
\hline Mean \pm Standard Deviation & $67.4 \pm 12.2$ & $65.4 \pm 11.5$ & $65.6 \pm 12$ & $65.5 \pm 11.7$ \\
\hline Median & 68.7 & 67.9 & 67.3 & 67.7 \\
\hline Minimum - Maximum & $19.2-92.2$ & $31-89$ & $23.3-91.4$ & $23.3-91.4$ \\
\hline n (\%) & $700(100 \%)$ & $415(100 \%)$ & $276(100 \%)$ & $691(100 \%)$ \\
\hline \multicolumn{5}{|c|}{ Baseline Left Ventricular Ejection Fraction (\%) } \\
\hline Mean \pm Standard Deviation & $27.7 \pm 10.5$ & $21 \pm 6.8$ & $30.3 \pm 11$ & $24.7 \pm 9.8$ \\
\hline Median & 25 & 20 & 30 & 25 \\
\hline Minimum - Maximum & $5-80$ & $6-35$ & $5-70$ & $5-70$ \\
\hline n (\%) & $691(98.7 \%)$ & $413(99.5 \%)$ & $273(98.9 \%)$ & $686(99.3 \%)$ \\
\hline \multicolumn{5}{|c|}{$\begin{array}{l}\text { Baseline New York Heart Association Classification } \\
(\mathrm{N}, \%)\end{array}$} \\
\hline Class I & $117(16.7 \%)$ & $0(0 \%)$ & $36(13 \%)$ & $36(5.2 \%)$ \\
\hline Class II & 297 (42.4\%) & $137(33 \%)$ & $102(37 \%)$ & $239(34.6 \%)$ \\
\hline Class III & $268(38.3 \%)$ & $237(57.1 \%)$ & $36(13 \%)$ & $273(39.5 \%)$ \\
\hline Class IV & $18(2.6 \%)$ & $4 \mathrm{I}(9.9 \%)$ & $3(1.1 \%)$ & $44(6.4 \%)$ \\
\hline Not Collected* & $0(0 \%)$ & $0(0 \%)$ & 99 (35.9\%) & 99 (14.3\%) \\
\hline Ischemic Cardiomyopathy & $488(69.7 \%)$ & $238(57.5 \%)$ & 155 (56.2\%) & $393(57.0 \%)$ \\
\hline Myocardial Infarction (N, \%) & $421(60.1 \%)$ & $193(46.6 \%)$ & $192(69.6 \%)$ & $385(55.8 \%)$ \\
\hline
\end{tabular}

N/A = not collected

*NYHA Class was only collected in the EMPIRIC Trial for patients with documented heart failure

The tachycardia detection and therapy parameters for the MIRACLE ICD and EMPIRIC patients included in the control arm were chosen at the discretion of the investigators and are referred to as "physician-tailored" parameters. [14]

\section{Data collection}

These data are collected at the 6 and 12 month scheduled visits and at unscheduled visits: cardiovascular adverse events, cardiovascular medications, VT/VF and SVT episodes, and patient diary.

Adverse events of syncope, near-syncope, and dizziness are collected between visits in a patient diary and evaluated for arrhythmogenic syncope, i.e. syncope or near syncope caused by a VT/VF or SVT episode, and symptomatic VT/VF events i.e. VT/VF events that result in the symptoms of near syncope or dizziness. All events in the PREPARE, EMPIRIC and MIRACLE ICD Trials will be adjudicated by the PREPARE Adverse Event Advisory Committee (AEAC) to identify arrhythmogenic syncope, near-syncope, and dizziness. The review includes the details surrounding each adverse event and the available EGM data for episodes occurring within 24 hours of the event. For the historical controls, where these adverse events were not as rigorously collected, the event will be adjudicated when the record indicated syncope, near-syncope, or dizziness in the narrative of an adverse event.

The differences in data collection methods between studies are always of concern when utilizing a historical control. There are two particular sources of bias when comparing the primary and key secondary endpoints in the PREPARE study to the historical control data: 1) adverse event collection and 2) incomplete adjudication of VT/VF episodes.

Patients in the PREPARE study are required to record adverse events of syncope, near-syncope, and dizziness in a patient diary, in an effort to increase the probability of collecting all occurrences of these events. By contrast, the MIRACLE ICD study relied on patient recall at study visits, and only system and procedure-related adverse events were collected in the EMPIRIC study. These methods of 
Trials 2006, 7:18

Table 2: PREPARE Study VT/VF Programming

\begin{tabular}{ll}
\hline ICD Parameter & Marquis/Maximo Family Programmed Value \\
\hline INDUCTION & \\
\hline VF Detection & ON \\
VFDI & $330 \mathrm{~ms}$ \\
VF NID & $30 / 40$ \\
VF RNID & $9 / 12$
\end{tabular}

\section{DUAL CHAMBER}

$\begin{array}{lc}\text { AF/AFI } & \text { ON } \\ \text { Sinus Tachcardia } & \text { ON } \\ \text { I:I VT-ST Boundary } & 66 \% \\ \text { Other I:I SVT } & \text { OFF } \\ \text { SVT Limit } & 300 \\ \text { Stability } & \text { OFF }\end{array}$

SINGLE CHAMBER

$\begin{array}{ll}\text { Wavelet } & \text { On, Match Threshold 70\% } \\ \text { Stability } & 40 \\ \text { Onset } & 88 \%\end{array}$

ALL

VF Detection

VFDI

VF NID

VF RNID

FVT

FVTDI

VT Detection

VTDI

VT NID

HR Timeout

VF Rx I-6 Status

VF Rx I-6 Energy

VF Rx I-4 Pathway

VF Rx 5-6 Pathway

VF Confirmation

FVT Rx I-6 Status

FVT Rx I Type

FVT Rx I Initial \# Pulses

FVT Rx I R-SI Interval

FVT Rx I \# sequence

FVT Rx I Smart Mode

FVT Rx 2-6 Type

FVT Rx 2-6 Energy

FVT Rx 2-5 Pathway

FVT Rx 6 Pathway

VT Rx I-6

Other Diagnostic and Therapy parameters
ON

$330 \mathrm{~ms}$

$30 / 40$

$9 / 12$

Via VF

$240 \mathrm{~ms}$

Monitor

$360 \mathrm{~ms}$

32

OFF

ON

30 or 35 J maximum output of the ICD

$A X->B$

$B->A X$

YES

ON

Burst

8

$88 \%$

I

OFF

$\mathrm{CV}$

30 to 35 J maximum output of ICD

$A X->B$

$B->A X$

OFF

Nominals collection in the historical control group likely led to underreporting of syncope related events, and therefore decreased the probability of arrhythmogenic syncopal or symptomatic VT/VF event discovery during these studies.
The expected consequence of this data limitation is that higher rates of arrhythmogenic syncope and symptomatic VT/VF events will be recorded in the PREPARE study. 
Not all episodes in the MIRACLE ICD study were originally adjudicated for the spontaneous/induced and true VT/true SVT classifications. To avoid the bias that would be incurred by dropping these episodes with missing information, these will be reviewed and classified by an internal Medtronic VT/VF scientist prior to the completion of the PREPARE study.

\section{Discussion \\ PREPARE programming strategies}

I) Strategies to reduce shocks for VT/VF

Olonger detection duration

$\mathrm{VF}$ initial beats to detect is set to 30 of 40 beats. At least $25 \%$ of ICD-detected VF is non-sustained VT/VF[15-17] The extended detection duration may decrease unnecessary shocks by allowing for spontaneous termination of non-sustained VT/VF that would otherwise be treated with nominal settings of shorter detection duration.

\section{OATP for FVTs 330-240 ms}

One sequence of ATP will be delivered for FVTs using the FVT via VF zone. Approximately $81 \%$ of ICD-detected VF (<320 ms) is monomorphic VT. Monomorphic VT can be pace terminated $75 \%$ of the time with one sequence of ATP. $[12,15,18,19]$ It is anticipated that programming a single sequence of ATP will reduce the use of shock therapy in this patient population, and thereby improve the patients' quality of life.

\section{Omaximum output for all VF therapy sequences and FVT sequence 2-6}

An initial high output shock may improve first shock success and thus reduce the likelihood of multiple shock episodes. In addition, the slightly longer charge time may allow more non-sustained VT/VF episodes to terminate before the first shock.

\section{2) Strategies to reduce shocks for SVTs Otachycardia detection at $330 \mathrm{~ms}$}

A treated cut-off of $320 \mathrm{~ms}$ has been used in several large primary prevention patient populations. $[7,14,20]$ In the latter two studies, investigators recommended a treatment cut-off between 330 and 350 ms. Detection of only tachyarrhythmias faster than 330 ms should reduce the inappropriate detection of SVTs.

\section{OVT monitor zone for slow VTs}

One recent study showed that only about $5 \%$ of patients with primary prevention indications experience monomorphic VT episodes slower than $360 \mathrm{~ms}$. [21] Setting a VT monitor zone between 360 and $330 \mathrm{~ms}$ is designed to reduce the inappropriate detection of SVTs, but still allow for identification of VT episodes below the therapy zone.

\section{Olonger detection duration}

VF initial beats to detect is set to 30 of 40 beats which may reduce detection of fast but nonsustained SVTs.

OPR logic (dual chamber) or Wavelet (single chamber)

SVT discriminators reduce detection of sustained SVTs of $300 \mathrm{~ms}$ (SVT Limit) or slower.

\section{Statistical analysis}

The primary endpoint is the difference in Morbidity Index incidence density between the study patients programmed to the PREPARE settings and the control population programmed to physician-tailored programming. The PREPARE programming strategy will be considered superior to the physician-tailored programming approach if a statistically significant reduction in the incidence density is demonstrated in the PREPARE group when compared to the historical control.

There were 347 shocked spontaneous VT/VF episodes, 4 events of arrhythmogenic syncope, and no episodes of untreated symptomatic sustained VT/VF in the 691 control population patients followed for 503 years (ID = 0.70). Assuming that the PREPARE programming strategy results in a $25 \%$ reduction in the number of shocked spontaneous VT/VF episodes, the rates of arrhythmogenic syncope and untreated symptomatic sustained VT/VF events remain the same, and the same number of years of follow-up, a total of 700 patients will provide at least $95 \%$ power for the primary hypothesis, tested at the two-sided significance level of 0.05 . Both the Morbidity Index ID and the secondary analysis of the Morbidity Tachycardia Index ID will be tested using the comparison of incidence rates test. [22]

\section{Summary}

The PREPARE study evaluates the ability of a pre-specified programming strategy to reduce VT/VF episode-related morbidity using a multi-center, prospective cohort design. The primary prevention population of 700 defibrillator patients with and without resynchronization therapy will be compared to 691 historical control patients. The VT/VF episode-related morbidity is assessed by a primary objective that indexes, for the number of years of follow-up, the total number of shocked spontaneous VT/VF episodes, untreated symptomatic sustained VT/VF episodes, and arrhythmogenic syncopal events.

The use of ICD and CRT+ICD devices in the primary prevention patient population has expanded dramatically over the last five years. Optimal programming for these patients has been investigated by other trials and understanding of these programming options continues to evolve. The programming choices available in the current devices enable additional specificity as it relates to the 
detection and treatment of ventricular arrhythmias. The PREPARE study results will increase the understanding of how to better develop programming strategies that positively impact primary prevention patients and their caregivers.

\section{Abbreviations}

AF/Afl: Atrial Fibrillation/Atrial Flutter

ATP: Anti-tachycardia Pacing

bpm: beats per minute

CRT: Cardiac Resynchronization Therapy

CRT + ICD: Cardiac Resynchronization Therapy with Implantable Cardioverter Defibrillator

EGM: Electrogram

EMPIRIC Evaluation of EMPIRIC Programming to Improve Patient Management

FVT: Fast Ventricular Tachycardia

FVTDI Fast Ventricular Tachycardia Detection Interval

HR Heart rate

ICD: Implantable Cardioverter Defibrillator

ID Incidence Density

MIRACLE ICD: Multicenter InSync ICD Randomized Clinical Evaluation Trial

ms: milliseconds

PREPARE Primary Prevention Parameters Evaluation

Rx Therapy

ST/Sinus Tach: Sinus Tachycardia

SVT: Supraventricular Tachycardia

VF: Ventricular Fibrillation

VFDI: Ventricular Fibrillation Detection Interval

VF NID: Ventricular Fibrillation Number of Intervals to Detect

VF RNID: Ventricular Fibrillation Number of Intervals to Redetect
VT: Ventricular Tachycardia

VTDI: Ventricular Tachycardia Detection Interval

VT NID: Ventricular Tachycardia Number of Intervals to Detect

\section{Competing interests}

1. In the past five years have you received reimbursements, fees, funding, or salary from an organization that may in any way gain or lose financially from the publication of this manuscript, either now or in the future?

Dr. Wilkoff: I am a consultant for Medtronic, St. Jude Medical and Guidant. Each of these companies manufactures ICDS.

Dr. Sterns: Medtronic Adverse Event Advisory Committee (AEAC), Medtronic

Lecturer

Dr. Williamson: Medtronic AEAC member

Dr. Wathen: Yes, Medtronic consultant

Holloman, Fieberg, Brown: Yes, salaried employees of Medtronic

2. Is such an organization financing this manuscript (including the article-processing charge)? If so, please specify.

Dr. Wilkoff, Dr. Stern, Dr. Williamson, Dr. Wathen: Holloman, Fieberg, Brown: Yes, Medtronic is paying for the processing fee of this article.

3. Do you hold any stocks or shares in an organization that may in any way gain or lose financially from the publication of this manuscript, either now or in the future? If so, please specify.

Dr. Wilkoff, Dr. Stern, Dr. Williamson, Dr. Wathen: No

Holloman, Fieberg, Brown: Yes, own Medtronic stock

4. Do you hold or are you currently applying for any patents relating to the content of the manuscript?

Dr. Wilkoff, Dr. Stern, Dr. Williamson, Dr. Wathen: Holloman, Fieberg, Brown: No

5. Have you received reimbursements, fees, funding, or salary from an organization that holds or has applied for 
patents relating to the content of the manuscript? If so, please specify.

Dr. Wilkoff, Dr. Stern, Dr. Williamson, Dr. Wathen: Holloman, Fieberg, Brown: No

6. Do you have any other financial competing interests? If so, please specify.

Dr. Wilkoff, Dr. Stern, Dr. Williamson, Dr. Wathen: Holloman, Fieberg, Brown: No

\section{Authors' contributions}

BLW is Principle Investigator for the study described in the manuscript. BLW made significant contributions to study design, drafting, revising the manuscript, and provided final approval of version to be published. RS served on the AEAC for the study described in the manuscript, participated in revising the manuscript and review prior to final approval of version to be published. BW served on the AEAC for the study described in the manuscript and participated in revising the manuscript and review prior to final approval of version to be published. MW served on the AEAC for the study described in the manuscript, provided input into specific aspects of study design and participated in review of the manuscript and review prior to final approval of version to be published. KH participated in the design of the study described in the manuscript, acquisition, analysis and interpretation of data for the study, drafting and revising the manuscript, and participated in review of the final version to be published. AF performed statistical analysis, participated in modification to the study design, drafting and revising the manuscript, and participated in review of the final version to be published. MB participated in development of the study concept, revisions to the manuscript and review of the final version to be published.

\section{Acknowledgements}

The PREPARE study was funded by Medtronic, Inc, Minneapolis, MN

\section{References}

I. AVID Investigators: A comparison of antiarrhythmic drug therapy with implantable defibrillators in patients resuscitated from near-fatal ventricular arrhythmias. NEJM 1997, 337:1576-1583.

2. Connolly S, Gent M, Roberts R, Dorian P, Roy D, Shelton R, Mitchel L, Green M, Klein G, O'Brien B, for the CIDS Investigators: Canadian Implantable Defibrillator Study (CIDS). Circulation 2000, I0I:1297-I302.

3. Kuck K, Cappato R, Siebels J, Ruppel R, for the CASH investigators: Randomized comparison of antiarrhythmic drug therapy with implantable defibrillators in patients resuscitated from cardiac arrest. The Cardiac Arrest Study Hamburg (CASH). Circulation 2000, 102:748-754.

4. Buxton AE, Lee KL, Fisher JD, Josephson M, Prystowsky EN, Hafley G: A Randomized Study of the Prevention of Sudden Death in Patients with Coronary Artery Disease. NEJM 1999, 341: 1882-1890.

5. Moss AJ, Hall WJ, Cannom DS, Daubert JP, Higgins SL, Klein H, Levine JH, Saksena S, Waldo AL, Wilber D, Brown MW, Heo M: Improved
Survival with an Implanted Defibrillator in Patients with Coronary Disease at High Risk for Ventricular Arrhythmia. NEJM 1999, 335: 1933-1940.

6. Moss AJ, Zareba W, Hall WJ, Klein H, Wilber DJ, Cannom DS, Daubert JP, Higgings SL, Brown M, Andrews ML: Prophylactic Implantation of a Defibrillator in Patients with Myocardial Infarction and Reduced Ejection Fraction. NEJM 2002, 346:877-883.

7. Bardy GH, Lee KL, Mark DB, Poole JE, Packer DL, Boineau R, Domanski M, Troutman C, Anderson J, Johnson G, McNulty SE, Clapp-Channing N, Davidson-Ray LD, Fraulo ES, Fishbein DP, Luceri RM, IP JH: Sudden Cardiac Death in Heart Failure Trial (SCD-HeFT) Investigators. N Engl J Med 2005, 352(20):2l 46.

8. Centers for Medicare \& Medicaid Services(CMS) Decision Memo for Coverage of Implantable Defibrillators. . January 27, 2005

9. Sears SF, Todaro JF, Urizar G, Lewis TS, Sirois B, Wallace R, Sotile W, Curtis $A B$, Conti JB: Assessing the psychosocial impact of the ICD: a national survey of implantable cardioverter defibrillator health care providers. PACE 2000, 23:1-6.

10. Luderitz B, Jung W, Deister A, Marneros A, Manz M: Patient acceptance of the implantable cardioverter defibrillator in ventricular tachyarrhythmias. PACE |999, 16(9):|8|5-2|.

II. Wilkoff BL, Ousdigian KT, Sterns LD, Wang Z], Wilson RD, Morgan JM, for the EMPIRIC Trial Investigators: A Comparison of Empiric to Physician-Tailored Programming of Implantable Cardioverter-Defibrillators : Results from the Prosepective Randomized Multicenter EMPIRIC Trial. in press.

12. Wathen MS, DeGroot PJ, Sweeney MO, Stark AJ, Otterness MF, Adkisson WO, Canby RC, Koroush K, Machado C, Rubenstein DS, Volosin K]: Prospective Randomized Multicenter Trial of Empirical Antitachycardia Pacing for Spontaneous Rapid Ventricular Tachycardia in Patients with Implantable Cardioverter-Defibrillators. Circulation 2004, I I 0:259|-2596.

13. Young JB, Abraham WT, Smith AL, Leon AR, Lieberman R, Wilkoff BL, Canby RC, Schroeder JS, Liem LB, Hall S, Wheelan K, The MIRACLE ICD Trial Investigators: Combined Cardiac Resynchronization and Implantable Cardioversion Defibrillation in Advanced Chronic Heart Failure. Journal of the American Medical Association 2003, 289(20):2685-272I.

14. Wilkoff BL, Hess M, Young J, Abraham WT: Differences in tachyarrhythmia detection and implantable cardioverter defibrillator therapy by primary or secondary prevention indication in cardiac resynchronization therapy patients. J Cardiovasc Electrophysio 2004, I 5:1002-1009.

15. Ousdigian K, Gillberg J, Koehler J, Stadler R, DeGroot P, Olson W, for the Worldwide GEM DR Investigators: How Often Is ICDDetected VF Actually Monomorphic VT? PACE 200I, 24:73I.

16. Gunderson B, Olson W, Brown T, Swerdlow C, and the GEM DRClinical Worldwide Investigators: Increasing the Number of Intervals for VF Detection Increases Implantable Cardioverter-Defibrillator Longevity. Circulation 2002, I06(19):II-322.

17. DeGroot P, Sharma V, Wathen M, Sweeney M, Chisner M, Machado C, Adkisson W, Koehler J, Stark A, for the PainFREE Rx Investigators: Implantable Cardioverter Defibrillator Detected Ventricular Fibrillation with Cycle Length $<240$ is Predominantly Fast Monomorphic Tachycardia. PACE 200I, 24:722.

18. Wathen M, Sweeney M, DeGroot P, Stark A, Koehler J, Chisner M, Machado D, Adkisson W: Shock Reduction Using Antitachycardia Pacing for Spontaneous Rapid Ventricular Tachycardias in Patients with Coronary Artery Disease. Circulation 2000, 104:769-80I.

19. Schaumann A, Muhlen F, Herse B, Gonska B, Kreuzer H: Empirical Versus Tested Anti-tachycardia Pacing in Implantable Cardioverter Defibrillators. Circulation 1998, 97:66-74.

20. Russo AM, Nayak H, Verdino R, et al.: Implantable cardioverter defibrillator events in patients with asymptomatic nonsustained ventricular tachycardia: is device implantation justified? Pacing Clin Electrophysiol 2003, 26( I 2):2289-2295.

2I. Ousdigian K, Wilkoff B, Morgan J, Sterns L, Wang Z, Wilson R: ICD Patients with a History of Spontaneous Sustained VT Have Different Outcomes Than Other Secondary or Primary Prevention Patients. Circulation 2005, I I 2(I 7):ii-374.

22. Rosner B: Fundamentals of Biostatistics. Fourth edition. Wadsworth Publishing Company; 1995. 